\title{
The effectiveness of financial intervention strategies for reducing caesarean section rates: a systematic review
}

\author{
Yushan Yu ${ }^{1,2,3+}$, Feili Lin ${ }^{1 \dagger}$, Weizhen Dong ${ }^{4}$, Haohan $\mathrm{Li}^{5}$, Xiangyang Zhang ${ }^{6 *}$ and Chun Chen ${ }^{1^{*}}$ (D)
}

\begin{abstract}
Background: The increasing trend of Caesarean section (CS) in childbirth has become a global public health challenge. Previous studies have proposed financial intervention strategies for reducing CS rates by limiting caesarean delivery on maternal request (CDMR). This study synthesizes such strategies while evaluating their effectiveness.

Methods: The sources of data for this study are Cochrane Library, PubMed, EMBASE, and CINAHL. The publication period included in this study is from January 1991 to November 2018. The financial intervention strategies are divide into two categories: healthcare provider interventions and patient interventions. Risk of Bias in Non-randomized Studies - of Interventions (ROBINS-I) was employed to assess the risk of bias of included studies. The outcome of each study was evaluated with Grades of Recommendation, Assessment, Development and Evaluation (GRADE) through the GRADEpro Guideline Development Tool software.
\end{abstract}

Results: Nine studies were included in this systematic review: five with high certainty evidence (HCE), three with moderate certainty evidence (MCE), and one with low certainty evidence (LCE). Of the nine studies, seven are centered on the effect of provider-side interventions. Three of the HCE studies found that the diagnosis-related group payment system, risk-adjusted capitation, and equalizing fee for both facilities and physicians were effective intervention strategies. One HCE and one MCE study showed that only equalizing facility fees between vaginal and CS deliveries in healthcare service settings had no significant effect on reducing the CS rate. The MCE study showed that case payment had a negative effect on reducing the CS rates. One LCE study revealed that the effect of a global budget system was uncertain, and one HCE and one MCE study focused on combining both provider and patient-side interventions. However, equalizing fees for vaginal and CS deliveries and a co-payment policy for CDMRs failed to reduce the CS rate.

Conclusions: The effectiveness of risk-adjusted payment methods appears promising and should be the subject of further research. Financial interventions should consider stakeholders' characteristics, especially the personal interests of doctors. Finally, high-quality randomized control trials and comparative studies on different financial intervention methods are needed to confirm or refute previous studies' outcomes.

Keywords: Financial intervention, Caesarean section rate, CDMR

\footnotetext{
*Correspondence: zxyanghero@126.com; chenchun408@126.com

${ }^{\dagger}$ Yushan Yu and Feili Lin contributed equally to this work.

${ }^{6}$ First Affiliated Hospital of Wenzhou Medical University, Wenzhou 325000,

China

${ }^{1}$ School of Public Health and Management, Wenzhou Medical University,

Tongren, Building 7B304, Wenzhou Medical University Chashan campus,

Wenzhou 325035, China

Full list of author information is available at the end of the article
}

(c) The Author(s). 2019 Open Access This article is distributed under the terms of the Creative Commons Attribution 4.0 International License (http://creativecommons.org/licenses/by/4.0/), which permits unrestricted use, distribution, and reproduction in any medium, provided you give appropriate credit to the original author(s) and the source, provide a link to the Creative Commons license, and indicate if changes were made. The Creative Commons Public Domain Dedication waiver (http://creativecommons.org/publicdomain/zero/1.0/) applies to the data made available in this article, unless otherwise stated. 


\section{Background}

The increasing trend of Caesarean section (CS) in childbirth has become a global public health challenge. Although the World Health Organization (WHO) is no longer recommending any specific CS rate, it has continuously warned that the rapid increase of CS rate should not be ignored, and has emphasized the need to avoid unnecessary CS worldwide [1]. In fact, that CS rate has increased from $19.5 \%(2000)$ to $27.2 \%$ (2014) in developed countries and from $13.1 \%$ (2000) to $20.9 \%$ (2014) in middle-income countries [2, 3]. Some less developed countries, such as Uganda and Kenya, are also experiencing a CS rate increase trend, although their growth rate is much slower than that of wealthier countries [4]. Unnecessary CS, or caesarean delivery on maternal request (CDMR) is the main cause of high CS rates [5]. The major concern with the increasing rates due to CDMR is that $\mathrm{CS}$ is associated with many short-term and long-term risks [6, 7] such as increased risk of asthma and obesity in children and increased risk of placenta previa and uterine rupture for mothers. Furthermore, high CS rates place a heavy burden on healthcare resources, which affects healthcare access equity $[8,9]$.

Therefore, different countries' governments and their respective healthcare sectors have developed and adopted various intervention strategies for containing and reducing CS rates, particularly by limiting the use of CDMR. Such intervention strategies include professional, financial, and regulatory ones. Healthcare authorities and managed care organizations have primarily explored the aspects of financial interventions to contain and reduce the CS rates by controlling unnecessary CS [10-14]. Financial interventions are external motivations that intend to change the behavior of the demand or the supply side through monetary incentives [15]. Some studies showed that financial interventions had a positive effect on promoting a variety of healthcare services, such as improving outcomes in outpatient behavioral treatments [16], enhancing warfarin adherence [17], maintaining smoking cessation [18], and increasing the utilization of vaccinations [15].

A CS is a service provided in medical care settings. In theory, financial intervention strategies could influence the behaviors of doctors and mothers and have a direct or indirect effect on the rate of CS deliveries. Recently, researchers found evidence that non-clinical interventions reduced the rate of unnecessary CS.

We identified seven related reviews published in the last 9 years [19-25], which addressed a range of non-clinical strategies intended to reduce CS births, including educational interventions [20, 21, 23, 25], organizational interventions [20, 21, 23, 24], regulatory interventions [21, 23], audits and feedback [19-21, 23], practice guidelines [20, 21, 23], and financial interventions [20, 21, 23]. Some financial interventions, such as fee equalizing and financial reimbursement strategies, were discussed; however, the findings were conflicting. Thus, we argue that it is urgent and critical to identify the effectiveness of various financial interventions in reducing CS rates, because financial incentives are a major driver in modern society irrespective of a country's income level.

Thus, the objectives of this systematic review are: (1) to determine the main financial intervention strategies developed, (2) to evaluate the effectiveness of these strategies, and (3) to synthesize relevant knowledge for policymakers to formulate financial interventions for reducing CS rates. Our review examines search strategies, study eligibility criteria, and the criteria for assessing the certainty of evidence.

\section{Method}

\section{Search strategy}

We conducted a systematic search of English language CS rate relevant articles in the following electronic databases: Cochrane Library (1991 to November 2018), MEDLINE/ PubMed (1948 to November 2018), EMBASE (1947 to November 2018), and CINAHL (1982 to November 2018) Additional file 1 . We first searched these electronic databases using different combinations of search terms as shown in Fig. 1. Then, we conducted an additional search by screening the reference lists from the selected literature.

\section{Inclusion criteria}

The inclusion criteria for this review consist of the following:

(a) Time range: Papers published between January 1991 to November 2018. This restriction was to ensure that they accurately represent financial interventions developed in recent decades.

(b) Types of studies: The included studies are randomized controlled trials, controlled clinical trials, cohort analytics (two groups: pre and post), cohort (one group pre + post: before and after), and interrupted time series (ITS) in which the intervention time was clearly defined, and there were at least three observations over time.

(c) Participants: The study participants are pregnant women and healthcare providers who work with expectant mothers. Studies on patients with particular conditions or specific risk factors (e.g., human immunodeficiency virus, pregnancy complications, preeclampsia, diabetes, obesity, hepatitis $B$, and herpes simplex virus) are excluded.

(d) Types of financial interventions: Financial interventions can be classified into two main types: provider and patient interventions (Fig. 1). The former includes fee-for-service, prepaid fee, capitation, provider salaried service, prospective payment, and provider financial penalty, while the 


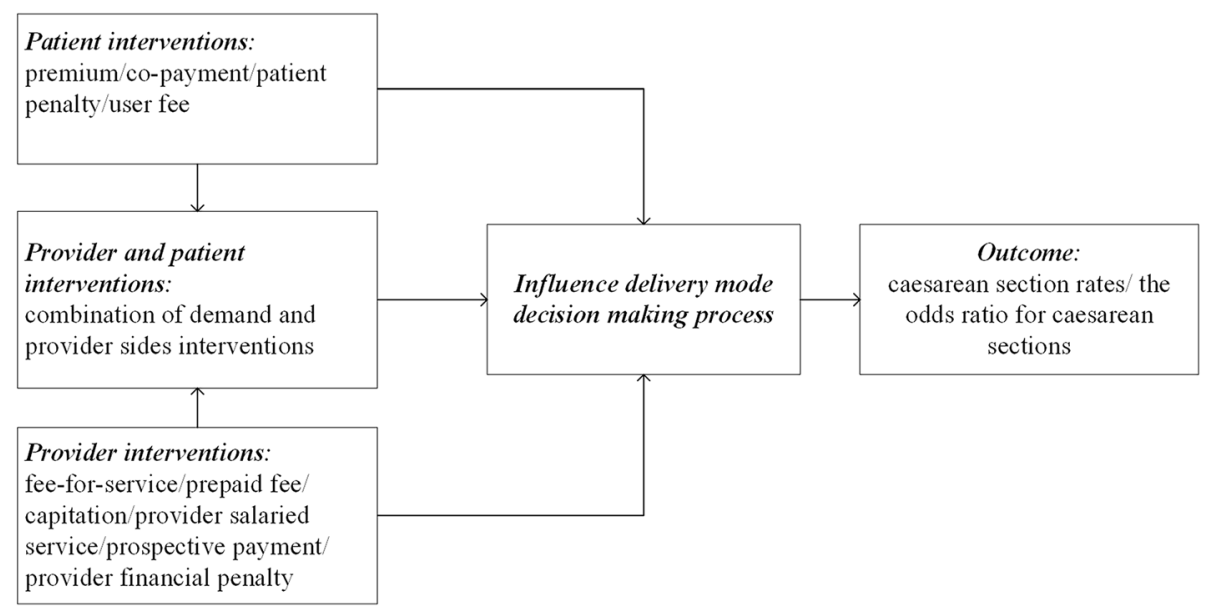

Fig. 1 Financial interventions and the utilization of caesarean sections

latter includes premium, co-payment, patient penalty, and user fees [26]. This study's focus is on the financial interventions that aim to reduce the CS rate.

(e) Types of outcomes: CS rates and the CS odds ratio are considered, while other outcomes are seen as useful secondary information. Studies that only reported other outcomes but no CS rates and the CS odds ratio are not included.

\section{Study selection and data extraction}

This study began with the selection of relevant publications' titles and abstracts based on the searches' keywords. Studies that met the inclusion criteria were identified. In the case of duplicate studies, the most relevant or most recent publication was included.

Data extraction was performed independently by the researchers using a self-designed data collection form containing the following information for each study: publication date, design, participant/data type, intervention, sample size, measures, results, statistics, and effect on CS rate (significant decrease, significant increase, no significant effect, and limited its increase). Primary authors were contacted for clarification when there was missing information on study design or intervention characteristics.

\section{Methodological quality}

Risk Of Bias In Non-randomized Studies - of Interventions (ROBINS-I) is a new tool for assessing the risk of bias in non-randomized studies for many kinds of organizational and public health interventions [27]. There are no randomized studies included in this systematic review. Thus, we used ROBINS-I to assess the risk of bias of each study. ROBINS-I was employed to assess the following aspects: a) confounders, b) selection of participants, c) classification of intervention, d) departure from intended interventions, e) missing data, f) measurement of outcomes, and g) selective reporting. Each part has five outcomes, namely, low risk of bias, moderate risk of bias, serious risk of bias, critical risk of bias, and no information [27]. The outcome of each study was evaluated with Grades of Recommendation, Assessment, Development and Evaluation (GRADE) using the GRADEpro Guideline Development Tool software [28], which categorized the quality or certainty of evidence into four levels: high, moderate, low, or very low.

\section{Results}

\section{Characteristics of the included studies}

Our search found 5,898 articles: 5,666 were rejected after the initial screening, 343 were beyond the period of this study, and 5,323 did not report the CS rate or CS odds ratio. From the remaining 232 articles and 16 articles identified from reference lists, 239 were removed because their study designs were not about aiming to reduce the CS rate. Finally, nine studies that met all of this study's criteria were included for the review (Fig. 2).

As shown in Table 1, there are no randomized controlled trials included in this study. The main design of included studies is ITS, and most studies were published in or after 2008. For the results of ROBINS-I, five studies had moderate risk of bias, three had serious risk of bias, and one had critical risk of bias. The results from GRADE are as follows: five studies were categorized as high certainty evidence, three studies as moderate certainty evidence, and another as low certainty evidence (Table 2). Seven focused on provider interventions, while two involved both provider and patient-side interventions (Table 3).

\section{Provider-side interventions}

Based on the assumption that a higher fee for CS could lead to an increase in CS abuse by healthcare providers, 


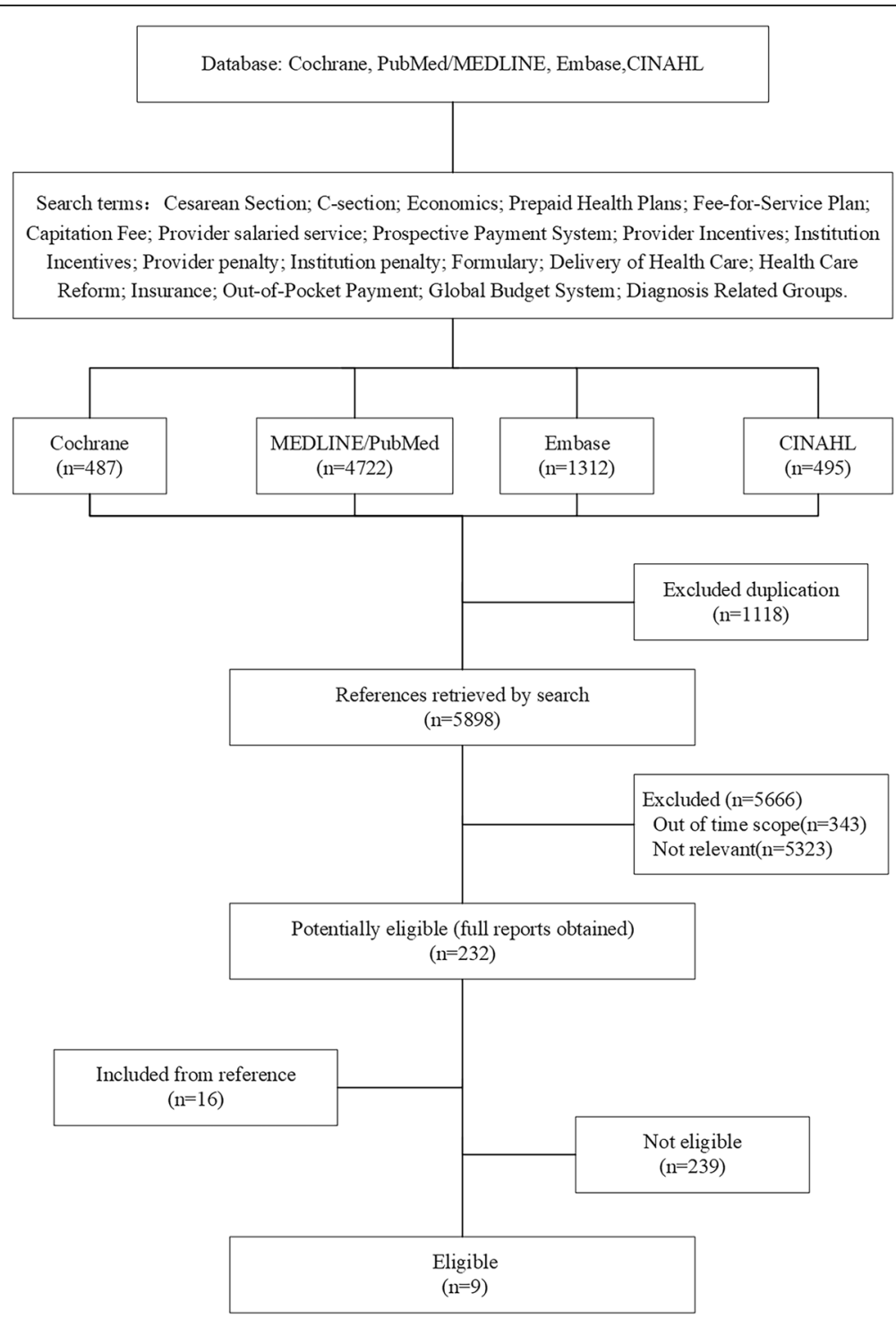

Fig. 2 Flow Diagram of Study Selection

three studies [29, 30, 37] reported on attempts at controlling CS rates through equalizing fees, including facility fees and professional fees. Facility fees are often charged in healthcare settings to cover operation costs [38], while professional fees are charged by doctors for medical services they provide to patients [39]. Keeler [29] and Lo [30] showed that fee-equalizing for facilities had no significant effect on reducing CS rates. In the first case, California Blue Cross increased the fee by 3\% for vaginal deliveries and reduced the fee by $18 \%$ for CS to decrease the CS rate in 1993 (moderate certainty evidence) [29]. In the second case, aiming to reduce the CS rate, the National Health Insurance of Taiwan raised the fee for vaginal birth after a CS (VBAC) to the level of CS since April 2003, following which all fees for vaginal deliveries were raised to the level of CS in May 2005 (high certainty evidence) [30]. It is worth noting that another high certainty evidence from Kozhimannil [37] showed that an equality fee intervention conducted by Minnesota's Medicaid Program, which raised both facility fees and professional fees, significantly decreased the CS rate.

Four of the studies examined the effect of payment reform for reducing the CS rate [31, 33, 35, 36]. In Taiwan, China, Liu et al. [33] evaluated the effectiveness of the hospital global budget system (GBS) reform to reduce the caesarean section rates in a tertiary hospital was uncertain in 2002 (low certainty evidence). In Henan, China, Liu et al. [36] described that payment reform from 2009 to 2011 that transforming a fee-for-service payment policy into a case payment policy has the opposite effect of increased the CS rate (moderate certainty 


\begin{tabular}{|c|c|c|c|c|c|c|c|c|c|}
\hline 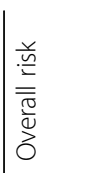 & 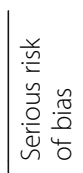 & 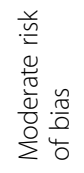 & 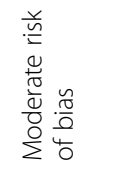 & 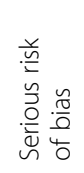 & 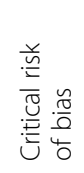 & 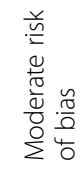 & 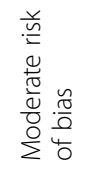 & 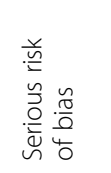 & 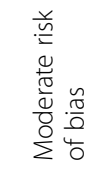 \\
\hline 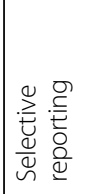 & $\begin{array}{l}\frac{n}{0} \\
\frac{0}{0} \\
4 \\
0 \\
. \frac{5}{2} \\
3 \\
0 \\
0\end{array}$ & 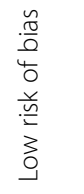 & 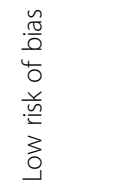 & $\begin{array}{l}\frac{n}{0} \\
\frac{.0}{0} \\
0 \\
.0 \\
.0 \\
0 \\
3 \\
0\end{array}$ & $\begin{array}{l}\frac{n}{0} \\
\frac{.0}{0} \\
0 \\
0 \\
\frac{1}{2} \\
3 \\
0 \\
0\end{array}$ & 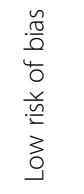 & 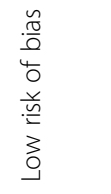 & 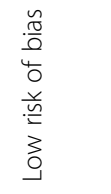 & 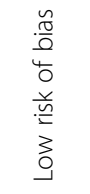 \\
\hline 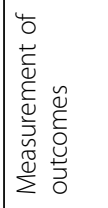 & 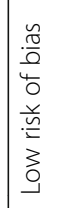 & $\begin{array}{l}\frac{.}{0} \\
\frac{0}{0} \\
\frac{0}{0} \\
\frac{.0}{0} \\
3 \\
0 \\
0\end{array}$ & $\begin{array}{l}\frac{n}{0} \\
\frac{.0}{0} \\
\frac{0}{0} \\
\frac{.}{3} \\
3 \\
3 \\
0\end{array}$ & $\begin{array}{l}\frac{n}{0} \\
\frac{0}{0} \\
40 \\
\frac{1}{0} \\
3 \\
0 \\
0\end{array}$ & $\begin{array}{l}\frac{n}{0} \\
\frac{0}{0} \\
40 \\
\frac{1}{0} \\
3 \\
3 \\
0\end{array}$ & 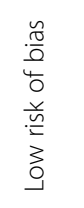 & 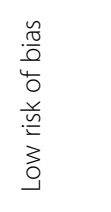 & $\begin{array}{l}\frac{n}{0} \\
\frac{.0}{0} \\
0 \\
\frac{1}{0} \\
3 \\
3 \\
0\end{array}$ & 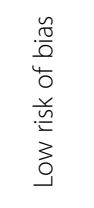 \\
\hline 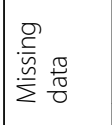 & 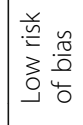 & 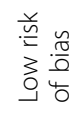 & 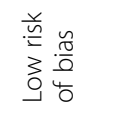 & 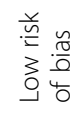 & 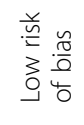 & 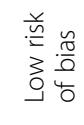 & 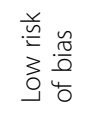 & 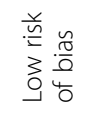 & 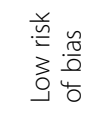 \\
\hline 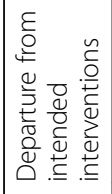 & 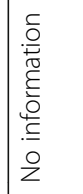 & 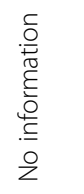 & 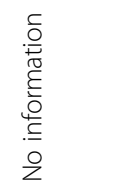 & 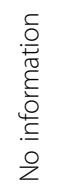 & 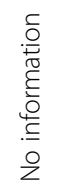 & 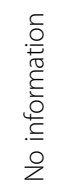 & 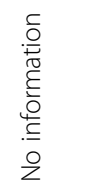 & 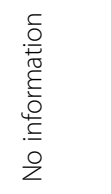 & 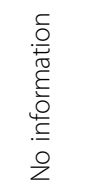 \\
\hline 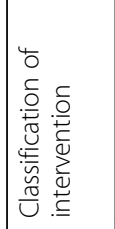 & 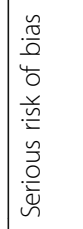 & 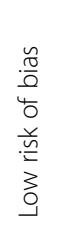 & 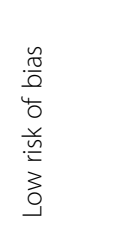 & 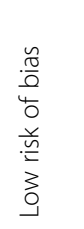 & $\begin{array}{l}\frac{n}{0} \\
\frac{.0}{0} \\
0 \\
\frac{1}{0} \\
\frac{0}{2} \\
3 \\
0\end{array}$ & 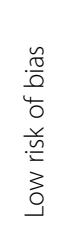 & 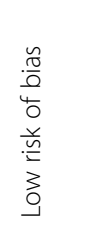 & 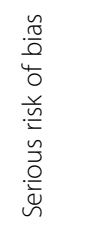 & $\begin{array}{l}0 \\
4 \\
0 \\
\frac{1}{3} \\
3 \\
3\end{array}$ \\
\hline 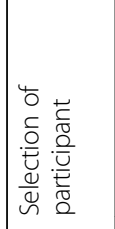 & 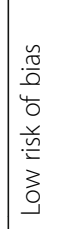 & 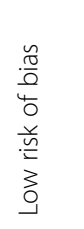 & 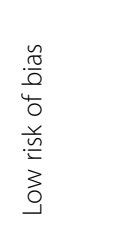 & 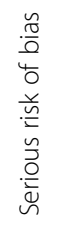 & 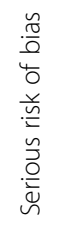 & 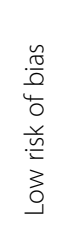 & 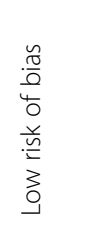 & 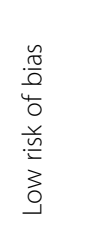 & $\begin{array}{l}\overline{0} \\
4 \\
0 \\
\frac{y}{2} \\
3 \\
0\end{array}$ \\
\hline 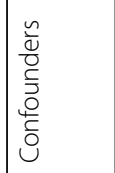 & 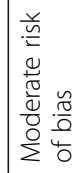 & 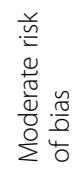 & 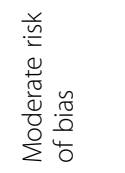 & 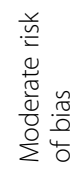 & 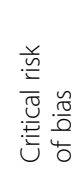 & 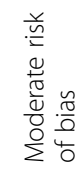 & 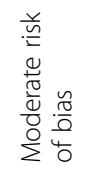 & 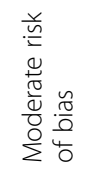 & 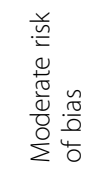 \\
\hline 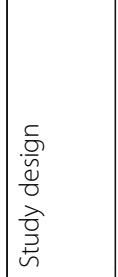 & 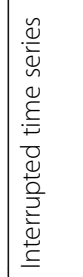 & 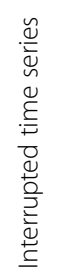 & 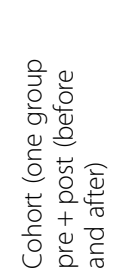 & 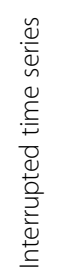 & 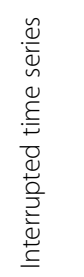 & 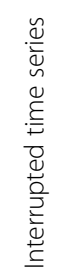 & 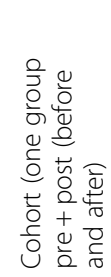 & 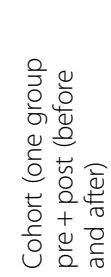 & 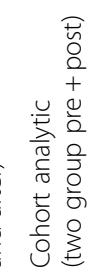 \\
\hline & 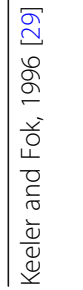 & 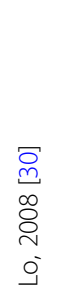 & 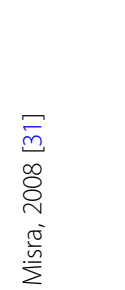 & 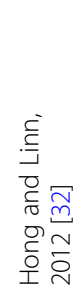 & 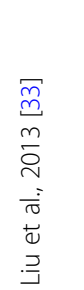 & 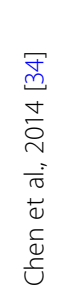 & 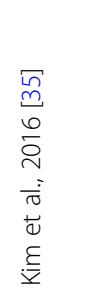 & 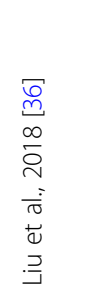 & 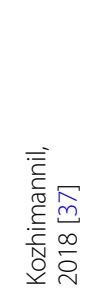 \\
\hline
\end{tabular}


Table 2 GRADE evidence of included studies

\begin{tabular}{|c|c|c|c|c|c|c|c|}
\hline \multirow[t]{2}{*}{ Author, year } & \multicolumn{6}{|l|}{ Certainty assessment } & \multirow{2}{*}{$\begin{array}{l}\text { Certainty } \\
\text { (GARDE) }\end{array}$} \\
\hline & Study design & Risk of bias & Inconsistency & Indirectness & Imprecision & Other considerations & \\
\hline \multirow[t]{2}{*}{ Keeler and Fok, 1996 [29] } & \multirow[t]{2}{*}{ Interrupted time series } & \multirow[t]{2}{*}{ serious } & \multirow[t]{2}{*}{ single study } & \multirow[t]{2}{*}{ not serious } & \multirow[t]{2}{*}{ not serious } & \multirow[t]{2}{*}{ none } & $\oplus \oplus \oplus \ominus$ \\
\hline & & & & & & & MODERATE \\
\hline \multirow[t]{2}{*}{ Lo, 2008 [30] } & \multirow[t]{2}{*}{ Interrupted time series } & \multirow[t]{2}{*}{ not serious } & \multirow[t]{2}{*}{ single study } & \multirow[t]{2}{*}{ not serious } & \multirow[t]{2}{*}{ not serious } & \multirow[t]{2}{*}{ none } & $\oplus \oplus \oplus \oplus$ \\
\hline & & & & & & & $\mathrm{HIGH}$ \\
\hline \multirow[t]{2}{*}{ Misra, 2008 [31] } & \multirow{2}{*}{$\begin{array}{l}\text { Cohort (one group } \\
\text { pre + post (before and after) }\end{array}$} & \multirow[t]{2}{*}{ not serious } & \multirow[t]{2}{*}{ single study } & \multirow[t]{2}{*}{ not serious } & \multirow[t]{2}{*}{ not serious } & \multirow[t]{2}{*}{ none } & $\oplus \oplus \oplus \oplus$ \\
\hline & & & & & & & $\mathrm{HIGH}$ \\
\hline \multirow{2}{*}{$\begin{array}{l}\text { Hong and Linn, } \\
2012 \text { [32] }\end{array}$} & \multirow[t]{2}{*}{ Interrupted time series } & \multirow[t]{2}{*}{ serious } & \multirow[t]{2}{*}{ single study } & \multirow[t]{2}{*}{ not serious } & \multirow[t]{2}{*}{ not serious } & \multirow[t]{2}{*}{ none } & $\oplus \oplus \oplus \ominus$ \\
\hline & & & & & & & MODERATE \\
\hline \multirow[t]{2}{*}{ Liu et al., 2013 [33] } & \multirow[t]{2}{*}{ Interrupted time series } & \multirow[t]{2}{*}{ critical serious } & \multirow[t]{2}{*}{ single study } & not serious & not serious & none & $\oplus \oplus \ominus \ominus$ \\
\hline & & & & & & & LOW \\
\hline Chen et al., 2014 [34] & Interrupted time series & not serious & single study & not serious & not serious & none & $\oplus \oplus \oplus \oplus$ \\
\hline & & & & & & & $\mathrm{HIGH}$ \\
\hline Kim et al., 2016 [35] & Cohort (one group & serious & single study & not serious & not serious & none & $\oplus \oplus \oplus \oplus$ \\
\hline & pre + post (before and after) & & & & & & $\mathrm{HIGH}$ \\
\hline Liu et al., 2018 [36] & Cohort (one group & serious & single study & not serious & not serious & none & $\oplus \oplus \oplus \ominus$ \\
\hline & and after) & & & & & & MODERATE \\
\hline Kozhimannil, 2018 [37] & Cohort analytic & not serious & single study & not serious & not serious & none & $\oplus \oplus \oplus \oplus$ \\
\hline & & & & & & & $\mathrm{HIGH}$ \\
\hline
\end{tabular}

evidence). However, two high-certainty studies revealed that risk-adjusted payment could be effective for controlling the CS rate [31, 35]. First, Misra [31] described the effect of provider intervention with risk-adjusted capitation for CS in Maryland, USA. In this high certainty of evidence study, capitation was charged monthly according to the applicants' health status, which could limit increases of the CS rate [31]. Second, Kim et al. [35] showed that a diagnosis-related group payment system for CS versus fee-for-service system for vaginal deliveries was effective in reducing the CS rate in Korea.

\section{Both provider and patient-side interventions}

Two studies [32, 34] examined a financial intervention strategy in which provider intervention was combined with patient intervention. In both studies, the providerside intervention involved equalizing fees, for example, the physicians in medical centers would receive a payment of $\$ 911$ to $\$ 1,132$ regardless of delivery mode (vaginal delivery, caesarean section with medical indications, or CDMR) [32, 34]. The intervention on the patient-side was a co-payment policy for elective CS. For example, physicians would receive a payment of $\$ 1,203$ for a CDMR in medical centers once the co-payment policy was implemented, which included a reimbursement of $\$ 506$ from the Nation Health Insurance and a co-payment of $\$ 697$ from the patients, respectively [32, 34]. However, these two studies (one was of high certainty and the other of moderate certainty) showed both provider and patientside incentives had no significant effect in reducing the CS rate.

\section{Discussion}

This systematic review examined the effectiveness of patient-side and provider-side financial intervention strategies in limiting unnecessary CS.

With regard to the patient-side intervention, two existing studies revealed that co-payment had no significant effect on reducing the CS rate. This was consistent with previous studies [40]. However, expectant mothers may influence the delivery mode. For instance, some may fear pain during labor, have a belief in the deteriorating quality of care during labor and vaginal birth [40,41], and be willing to pay for CS [32]. However, doctors play a more important role than mothers do in the selection of delivery mode [42, 43], and mothers' preference of delivery mode is unlikely to be a major driver of high CS rates [40]. Thus, we do not recommend a co-payment policy to reduce CS rates without strong evidence supporting its effectiveness.

With regard to provider-side interventions, previous studies show that financial interventions could influence the behavior of physicians by promoting antibiotic prescribing practices [44], improving the provision of necessary healthcare [45], and improving clinical care quality [46]. Theoretically, financial incentive strategies could 


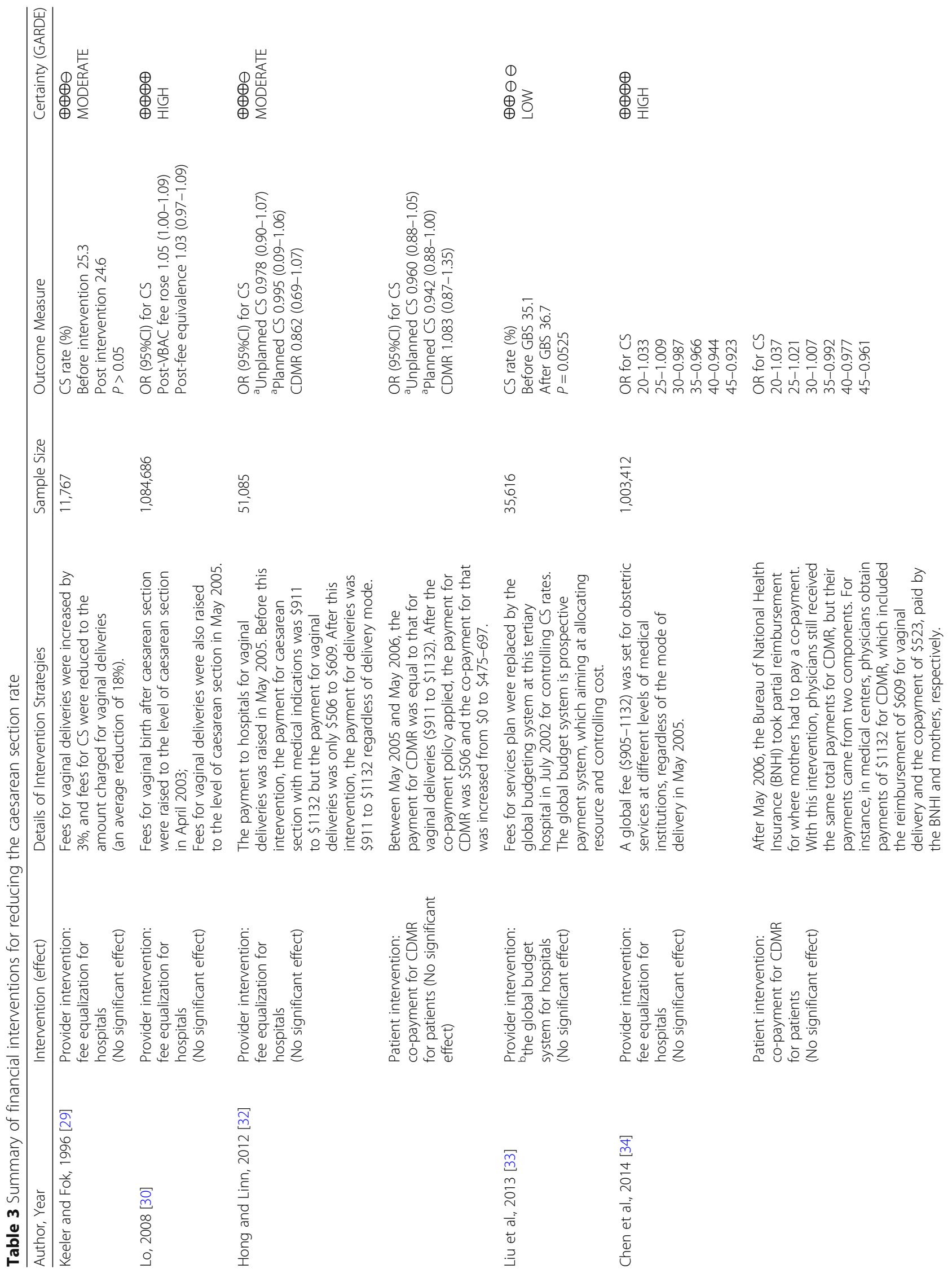




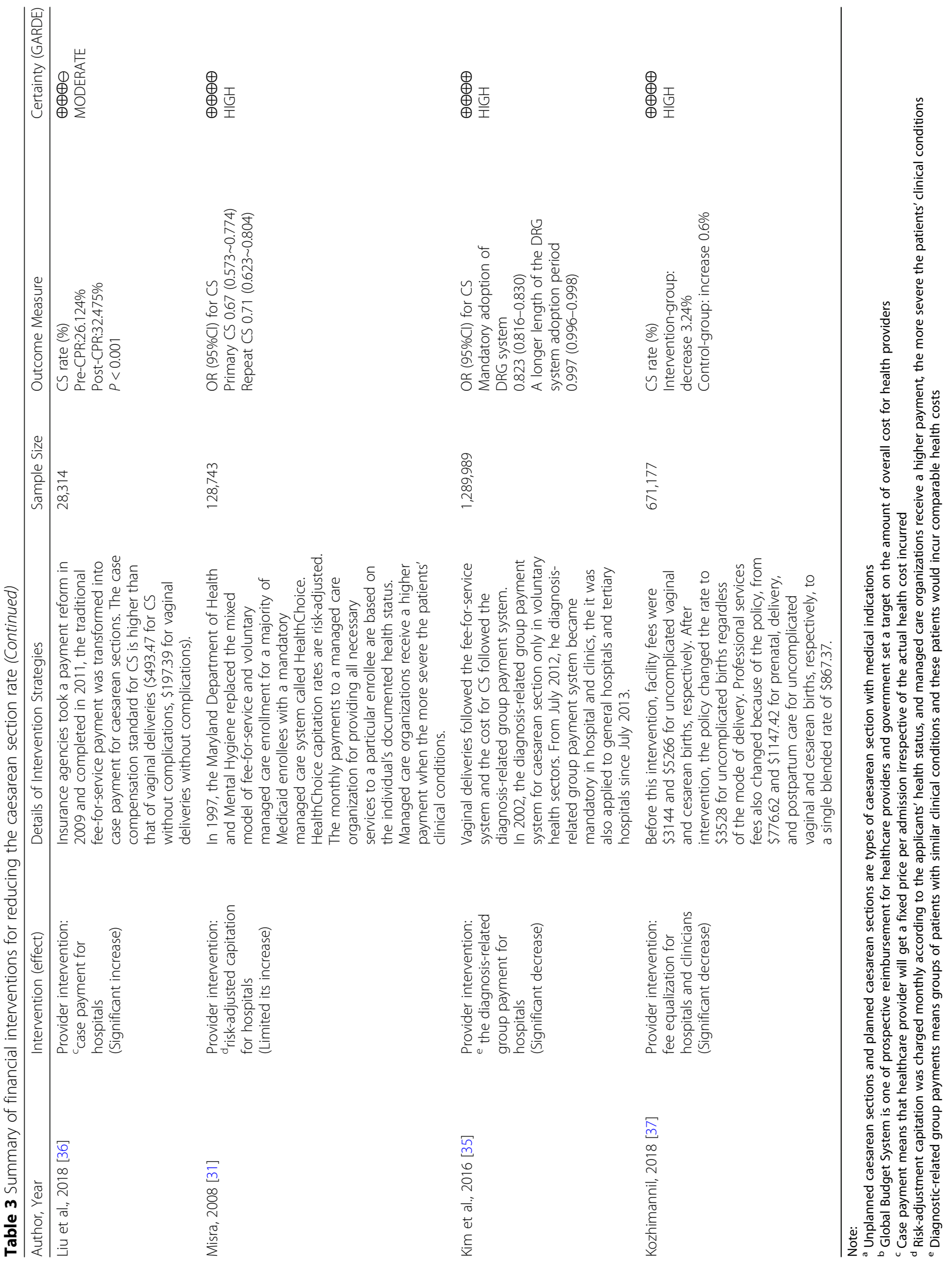


be effective in reducing the CS rate, especially the rate of CDMR. However, this intervention was not as effective as was expected.

Financial incentives for provider-side intervention in this systematic review comprise equalizing fees, case payment, national healthcare policy of the GBS, diagnosisrelated group payment system for CS, and risk-adjusted capitation for CS.

Simply equalizing fees for facilities was not effective in the existing literature for a few possible reasons: (1) CS is regarded as defensive medicine for avoiding medical lawsuits [47]; (2) the medical professionals' demand for leisure was positively associated with CS utilization because performing a CS is faster than a vaginal delivery, resulting in more leisure time for medical care providers $[48,49]$; and (3) lack of guidance from behavioral psychology and behavioral economics resulted in the creation of an ineffective financial intervention strategy [50]. In short, a simple economic incentive intervention appears to be less effective in influencing physicians' delivery mode decision than expected. Evidence from Taiwan, China suggested that the method of equalizing fees did not work at all. Medical resource consumption of a CS is generally higher than that of a vaginal delivery. However, even once the price of a vaginal delivery was raised to the level of a CS [32, 34], it still had no significant effect in reducing the CS rate. Therefore, equalizing fees for facilities alone did not appear to reduce the CS rate.

Evidence from Henan, China showed that case payment was not effective for reducing the CS rate, which could relate to unreasonable case compensation standards for CS (\$493.47), which is much higher than vaginal delivery (\$197.39) [36]. Thus, the healthcare setting and physicians may prefer CS over vaginal deliveries for financial reasons [51].

However, it appears that the risk-adjusted payment methods such as a diagnosis-related group payment system for CS and a risk-adjusted capitation for CS were effective in controlling the CS rate [31,33]. The potential reason is that a risk-adjusted payment system introduces competition among healthcare service providers [52]. The risk-adjusted price is based on competitive forces with other hospitals [53], with the healthcare setting receiving a risk-adjusted payment. Moreover, hospitals and clinics will incur a significant loss if physicians perform unnecessary medical care services such as CDMR [54, 55]. In addition, since a risk-adjusted payment system functions to improve cost management of the hospital [56], it will be advantageous to train and educate physicians to provide only medically necessary services.

Additionally, the unprecedented rapid increase of CS utilization rates is multifactorial. It includes behavioral, psychosocial, organizational, and financial factors of women, families, healthcare professionals, and healthcare organizations and systems [20]. Many related stakeholders influence the effectiveness of interventions. Working with different intervention priorities and interests poses a barrier for effective intervention implementation. Interventions that are single-component or that address the concerns/needs of one of the stakeholders without considering the others are not ideal and are more likely to fail. Therefore, policymakers must consider the interests of all stakeholders. In other words, based on the perspective of multi-interest groups, policymakers can find a "sensitive and cost-effective point" to reduce irrational CS utilization, and develop and implement corresponding strategies to guarantee the effectiveness of the financial intervention. The high certainty evidence from Minnesota's Medicaid Program is a good example of how an intervention policy that takes into account the personal interests of doctors by equalizing fees for both facilities and physicians could significantly decrease the CS rate [37].

\section{Limitations and strengths of the review}

We believe this is the first global study to focus on the effectiveness of various financial intervention strategies in reducing unnecessary CS. There are several limitations to the interpretation of our findings. First, existing studies are sparse and limited. Because there are too few studies in each sub-intervention group, as well as the diversity study design among sub-groups, we were unable to undertake the sub-analyses as we had planned. Second, lack of direct comparative studies made it difficult for us to point out which could be the best financial interventions for reducing the CS rate. Third, although all the studies were about reducing unnecessary CS, the clinical data about the appropriateness of the CS conducted were unavailable. Therefore, there is no basis for us to distinguish and assess whether the reduced number of CS were all unnecessary ones. Fourth, the impact of financial interventions on reducing CS is quite complex. Thus, differences in the studies may have resulted from unaccounted for differences in countries, policy environments, target population groups, and variables measured.

Future research using randomized controlled designs or fixed effect modeling longitudinal studies can provide more robust predictions regarding the effect of financial interventions on reducing unnecessary CS. Finally, the systematic review methodology and use of the ROBINS-I tool are the strengths of the review.

\section{Conclusions}

Although there is still a paucity of high-quality research on this topic, based on current evidence, we can draw three conclusions. First, although we can't draw the conclusion that the risk-adjusted payment methods such as a diagnosis-related group payment system for CS and a 
risk-adjusted capitation for CS are effective provider-side interventions. However, the effectiveness of these riskadjusted payment methods looks promising; thus, strong evidence is needed for proving that the provider-side intervention could be considered and is effective. Second, financial interventions should take stakeholders' characteristics into account, especially doctors' personal interests. Third, high quality RCT data and direct comparative studies on different financial interventions in the future could confirm or refute the outcomes of the existing research.

\section{Additional file}

Additional file 1: Full electronic search strategy used in the systematic review; We conducted a systematic search of English language CS rate relevant articles following this search strategy. (DOCX $21 \mathrm{~kb}$ )

\section{Abbreviations}

CDMR: Caesarean delivery on maternal request; CS: Cesarean section; GRADE: Grades of Recommendation, Assessment, Development, and Evaluation; HCE: Certainty evidence; ITS: Interrupted time series; LCE: Low certainty evidence; MCE: Moderate certainty evidence; RCT: Randomized control trial; ROBINS-I: Risk of Bias in Non-randomized Studies - of Interventions; VBAC: Vaginal birth after a cesarean section; WHO: World Health Organization

\section{Acknowledgements}

The authors thank Hongpo Yin and Yanjun Yang for their valuable assistance.

\section{Authors' contributions}

YSY and FLL collected the data and wrote the first draft of the paper. XYZ and $C C$ designed the original version of PLANS. CC, WD, and HHL conceived the study. All authors contributed to writing the paper. All authors read and approved the final manuscript.

\section{Funding}

This work was supported by the National Natural Science Foundation of China [71603187]; Zhejiang Provincial Natural Science Foundation [LQ16G030011]; and the Key Social Science Project for University Teachers of Zhejiang, China [2014QN005]. The funders played no role in the design of the study and collection, analysis, and interpretation of data and in writing the manuscript.

\section{Availability of data and materials}

The authors confirm that all data is contained within the manuscript and its additional files.

\section{Ethics approval and consent to participate}

Not applicable.

\section{Consent for publication}

Not applicable.

\section{Competing interests}

The authors declare that they have no competing interests.

\section{Author details}

${ }^{1}$ School of Public Health and Management, Wenzhou Medical University, Tongren, Building 7B304, Wenzhou Medical University Chashan campus, Wenzhou 325035, China. 'Department of Scientific Research and Education, Cancer Hospital, Chinese Academy of Science, Hefei 230031, Hefei, China. ${ }^{3}$ Department of Preventive Medicine, Medical School of Shihezi University, Shihezi 832003, China. ${ }^{4}$ Department of Sociology and Legal Studies, University of Waterloo, 200 University Avenue West, Waterloo, Ontario N2L 3G1, Canada. ${ }^{5}$ Zhongshan Hospital Fudan University, Shanghai 200032,
China. ${ }^{6}$ First Affiliated Hospital of Wenzhou Medical University, Wenzhou 325000, China

Received: 22 July 2018 Accepted: 30 June 2019

Published online: 09 August 2019

\section{References}

1. Betran AP, Torloni MR, Zhang JJ, Gulmezoglu AM. Section WHOWGoC: WHO statement on caesarean section rates. BJOG. 2016;123(5):667-70.

2. Betran AP, Ye J, Moller AB, Zhang J, Gulmezoglu AM, Torloni MR. The increasing trend in caesarean section rates: global, regional and National Estimates: 1990-2014. PLoS One. 2016;11(2):e0148343.

3. Ye J, Zhang J, Mikolajczyk R, Torloni MR, Gulmezoglu AM, Betran AP. Association between rates of caesarean section and maternal and neonatal mortality in the 21st century: a worldwide population-based ecological study with longitudinal data. BJOG. 2016;123(5):745-53.

4. Vogel JP, Betran AP, Vindevoghel N, Souza JP, Torloni MR, Zhang J, Tuncalp $\mathrm{O}$, Mori R, Morisaki N, Ortiz-Panozo E, et al. Use of the Robson classification to assess caesarean section trends in 21 countries: a secondary analysis of two WHO multicountry surveys. Lancet Global Health. 2015;3(5):E260-70.

5. Boerma T, Ronsmans C, Melesse DY, Barros AJD, Barros FC, Juan L, Moller $A B$, Say $L$, Hosseinpoor AR, Yi M, et al. Global epidemiology of use of and disparities in caesarean sections. Lancet. 2018;392(10155):1341-8.

6. Sandall J, Tribe RM, Avery L, Mola G, Visser GH, Homer CS, Gibbons D, Kelly NM, Kennedy HP, Kidanto H, et al. Short-term and long-term effects of caesarean section on the health of women and children. Lancet. 2018; 392(10155):1349-57.

7. Keag OE, Norman JE, Stock SJ. Long-term risks and benefits associated with cesarean delivery for mother, baby, and subsequent pregnancies: systematic review and meta-analysis. PLoS Med. 2018;15(1):e1002494.

8. Gibbons L, Belizán JM, Lauer JA, Betrán AP, Merialdi M, Althabe F. The global numbers and costs of additionally needed and unnecessary caesarean sections performed per year: overuse as a barrier to universal coverage; 2010

9. Boatin AA, Schlotheuber A, Betran AP, Moller AB, Barros AJD, Boerma T, Torloni MR, Victora CG, Hosseinpoor AR. Within country inequalities in caesarean section rates: observational study of 72 low and middle income countries. BMJ. 2018;360:k55.

10. Strategies for lowering C-section rates [https://www.managedhealth careexecutive.com/health-law-policy/strategies-lowering-c-section-rates] Accessed 24 Feb 2019.

11. Grant D. Physician financial incentives and cesarean delivery: new conclusions from the healthcare cost and utilization project. J Health Econ. 2009:28(1):244-50.

12. Kozhimannil KB, Law MR, Virnig BA Cesarean Delivery Rates Vary 10-Fold Among US Hospitals; Reducing Variation May Address Quality, Cost Issues. Health Aff (Millwood). 2013;32(3):527-35. https://www.ncbi.nlm.nih.gov/ pmc/articles/PMC3615450/.

13. Birthing A Movement To Reduce Unnecessary C-Sections: An Update From California [https://www.healthaffairs.org/do/10.1377/hblog20171 031.709216/full/]. Accessed 24 Feb 2019.

14. The Push to Reduce Unnecessary C-Sections [https://www.ourbodies ourselves.org/2016/05/push-to-reduce-unnecessary-c-sections/]. Accessed 24 Feb 2019.

15. Ndiaye SM, Hopkins DP, Shefer AM, Hinman AR, Briss PA, Rodewald L, Willis B. Task force on community preventive S: interventions to improve influenza, pneumococcal polysaccharide, and hepatitis B vaccination coverage among high-risk adults: a systematic review. Am J Prev Med. 2005;28(5 Suppl):248-79.

16. Higgins ST, Budney AJ, Bickel WK, Foerg FE, Donham R, Badger GJ. Incentives improve outcome in outpatient behavioral treatment of cocaine dependence. Arch Gen Psychiatry. 1994;51(7):568-76.

17. Volpp KG, Loewenstein G, Troxel AB, Doshi J, Price M, Laskin M, Kimmel SE. A test of financial incentives to improve warfarin adherence. BMC Health Serv Res. 2008:8:272

18. Halpern SD, French B, Small DS, Saulsgiver K, Harhay MO, AudrainMcGovern J, Loewenstein G, Brennan TA, Asch DA, Volpp KG. Randomized trial of four financial-incentive programs for smoking cessation. N Engl J Med. 2015;372(22):2108-17.

19. Boatin AA, Cullinane F, Torloni MR, Betran AP. Audit and feedback using the Robson classification to reduce caesarean section rates: a systematic review. BJOG. 2018;125(1):36-42. 
20. Betran AP, Temmerman M, Kingdon C, Mohiddin A, Opiyo N, Torloni MR, Zhang J, Musana O, Wanyonyi SZ, Gulmezoglu AM, et al. Interventions to reduce unnecessary caesarean sections in healthy women and babies. Lancet. 2018;392(10155):1358-68.

21. Chen I, Opiyo N, Tavender E, Mortazhejri S, Rader T, Petkovic J, Yogasingam S, Taljaard M, Agarwal S, Laopaiboon M, et al. Non-clinical interventions for reducing unnecessary caesarean section. Cochrane Database Syst Rev. 2018;9:CD005528.

22. Kingdon C, Downe S, Betran AP. Non-clinical interventions to reduce unnecessary caesarean section targeted at organisations, facilities and systems: systematic review of qualitative studies. PLoS One. 2018;13(9): e0203274.

23. Khunpradit S, Tavender E, Lumbiganon P, Laopaiboon M, Wasiak J, Gruen RL. Non-clinical interventions for reducing unnecessary caesarean section. Cochrane Database Syst Rev. 2011;(6):CD005528. https://doi.org/10.1 002/14651858.CD005528.pub2.

24. Hutchinson AM, Nagle C, Kent B, Bick D, Lindberg R. Organisational interventions designed to reduce caesarean section rates: a systematic review protocol. BMJ Open. 2018;8(7):e021120.

25. Kingdon C, Downe S, Betran AP. Women's and communities' views of targeted educational interventions to reduce unnecessary caesarean section: a qualitative evidence synthesis. Reprod Health. 2018;15(1):130.

26. The Data Collection Checklist. [http://epoc.cochrane.org/]. Accessed 24 Feb 2019.

27. Sterne JA, Hernan MA, Reeves BC, Savovic J, Berkman ND, Viswanathan M, Henry D, Altman DG, Ansari MT, Boutron I, et al. ROBINS-l: a tool for assessing risk of bias in non-randomised studies of interventions. BMJ. 2016:355:i4919.

28. GRADE your evidence and improve your guideline development in health care. [https://gradepro.org/]. Accessed 24 Feb 2019.

29. Keeler EB, Fok T. Equalizing physician fees had little effect on cesarean rates. Med Care Res Rev. 1996:53(4):465-71.

30. Lo JC. Financial incentives do not always work: an example of cesarean sections in Taiwan. Health Policy. 2008:88(1):121-9.

31. Misra A. Impact of the HealthChoice program on cesarean section and vaginal birth after $\mathrm{C}$-section deliveries: a retrospective analysis. Matern Child Health J. 2008;12(2):266-74.

32. Hong YC, Linn GC. Financial incentives and use of cesarean delivery: Taiwan birth data 2003 to 2007. Am J Manag Care. 2012;18(1):e35-41.

33. Liu CM, Lin YJ, Su YY, Chang SD, Cheng PJ. Impact of health policy based on the self-management program on cesarean section rate at a tertiary hospital in Taiwan. J Formos Med Assoc. 2013;112(2):93-8.

34. Chen CS, Liu TC, Chen B, Lin CL. The failure of financial incentive? The seemingly inexorable rise of cesarean section. Soc Sci Med. 2014;101:47-51.

35. Kim SJ, Han KT, Kim SJ, Park EC, Park HK. Impact of a diagnosis-related group payment system on cesarean section in Korea. Health Policy. 2016;120(6):596-603.

36. Liu S, Wang J, Zhang L, Zhang X. Caesarean section rate and cost control effectiveness of case payment reform in the new cooperative medical scheme for delivery: evidence from Xi County, China. BMC Pregnancy Childbirth. 2018;18(1):66.

37. Kozhimannil KB, Graves AJ, Ecklund AM, Shah N, Aggarwal R, Snowden JM. Cesarean delivery rates and costs of childbirth in a state Medicaid program after implementation of a blended payment policy. Med Care. 2018;56(8):658-64

38. Facility Fee Reporting. [https://www.doh.wa.gov/DataandStatisticalReports/ HealthcareinWashington/HospitalandPatientData/HospitalFinancialData/ HospitalFacilityFees]. Accessed 24 Feb 2019.

39. Health Care Services: Professional Fees. [https://policy.ucop.edu/doc/341 0233/AM-H214-7]. Accessed 24 Feb 2019.

40. Long Q, Kingdon C, Yang F, Renecle MD, Jahanfar S, Bohren MA, Betran AP. Prevalence of and reasons for women's, family members', and health professionals' preferences for cesarean section in China: a mixed-methods systematic review. PLoS Med. 2018;15(10):e1002672.

41. Handelzalts JE, Fisher S, Lurie S, Shalev A, Golan A, Sadan O. Personality, fear of childbirth and cesarean delivery on demand. Acta Obstet Gynecol Scand. 2012;91(1):16-21.

42. Ji H, Jiang H, Yang L, Qian X, Tang S. Factors contributing to the rapid rise of caesarean section: a prospective study of primiparous Chinese women in Shanghai. BMJ Open. 2015;5(11):e008994.
43. Deng W, Klemetti R, Long Q, Wu Z, Duan C, Zhang WH, Ronsmans C, Zhang Y, Hemminki E. Cesarean section in Shanghai: women's or healthcare provider's preferences? BMC Pregnancy Childbirth. 2014;14:285.

44. Yip W, Powell-Jackson T, Chen W, Hu M, Fe E, Hu M, Jian W, Lu M, Han W, Hsiao WC. Capitation combined with pay-for-performance improves antibiotic prescribing practices in rural China. Health Aff (Project Hope). 2014;33(3):502-10.

45. Chen CC, Cheng SH. Does pay-for-performance benefit patients with multiple chronic conditions? Evidence from a universal coverage health care system. Health Policy Plan. 2016;31(1):83-90.

46. Peabody J, Shimkhada R, Quimbo S, Florentino J, Bacate M, McCulloch CE, Solon O. Financial incentives and measurement improved physicians' quality of care in the Philippines. Health Aff (Project Hope). 2011;30(4):773-81.

47. Vimercati A, Greco P, Kardashi A, Rossi C, Loizzi V, Scioscia M, Loverro G. Choice of cesarean section and perception of legal pressure. J Perinat Med. 2000;28(2):111-7.

48. Costa i Font J. Do incentives, complexity and the demand for leisure explain caesarean-section deliveries? Int J Soc Econ. 2009;36(9):906-15

49. Brown HS 3rd. Physician demand for leisure: implications for cesarean section rates. J Health Econ. 1996;15(2):233-42.

50. Lapointe-Shaw L, Mamdani M, Luo J, Austin PC, Ivers NM, Redelmeier DA, Bell CM. Effectiveness of a financial incentive to physicians for timely followup after hospital discharge: a population-based time series analysis. CMAJ. 2017:189(39):E1224-9.

51. Hellerstein S, Feldman S, Duan T. China's $50 \%$ caesarean delivery rate: is it too high? BJOG. 2015;122(2):160-4.

52. Quentin W, Scheller-Kreinsen D, Blumel M, Geissler A, Busse R. Hospital payment based on diagnosis-related groups differs in Europe and holds lessons for the United States. Health Aff (Project Hope). 2013;32(4):713-23.

53. Antioch KM, Walsh MK. Risk-adjusted capitation funding models for chronic disease in Australia: alternatives to casemix funding. Eur J Health Econ. 2002;3(2):83-93.

54. Grytten J, Monkerud L, Hagen TP, Sorensen R, Eskild A, Skau I. The impact of hospital revenue on the increase in caesarean sections in Norway. A pane data analysis of hospitals 1976-2005. BMC Health Serv Res. 2011;11:267.

55. Mougeot M, Naegelen F. Supply-side risk adjustment and outlier payment policy. J Health Econ. 2008;27(5):1196-200.

56. Zeng $Y$, He AJ, Lin P, Sun Z, Fang Y. Developing case-mix standards with the diagnosis-related groups for payment reforms and hospital management in China: a case study in Xiamen city. Int J Healthcare. 2016;2(1):102-10.

\section{Publisher's Note}

Springer Nature remains neutral with regard to jurisdictional claims in published maps and institutional affiliations.

Ready to submit your research? Choose BMC and benefit from

- fast, convenient online submission

- thorough peer review by experienced researchers in your field

- rapid publication on acceptance

- support for research data, including large and complex data types

- gold Open Access which fosters wider collaboration and increased citations

- maximum visibility for your research: over $100 \mathrm{M}$ website views per year

At $\mathrm{BMC}$, research is always in progress.

Learn more biomedcentral.com/submission 